\title{
Video Article \\ Recapitulating Suckling-to-Weaning Transition In Vitro using Fetal Intestinal Organoids
}

\author{
Tânia Martins Garcia ${ }^{1}$, Marit Navis ${ }^{1}$, Manon E. Wildenberg ${ }^{1}$, Ruurd M. van Elburg ${ }^{2}$, Vanesa Muncan ${ }^{1}$ \\ ${ }^{1}$ Department of Gastroenterology and Hepatology, Tytgat Institute for Intestinal and Liver Research, Amsterdam UMC, AG\&M, University of Amsterdam \\ ${ }^{2}$ Department of Pediatrics, Amsterdam UMC, University of Amsterdam
}

Correspondence to: Vanesa Muncan at v.muncan@amsterdamumc.nl

URL: https://www.jove.com/video/60470

DOI: doi: $10.3791 / 60470$

Keywords: Developmental Biology, Issue 153, intestinal organoids, suckling-to-weaning transition, fetal intestine, gut maturation, brush border enzymes, in vitro maturation

Date Published: $11 / 15 / 2019$

Citation: Garcia, T.M., Navis, M., Wildenberg, M.E., van Elburg, R.M., Muncan, V. Recapitulating Suckling-to-Weaning Transition In Vitro using Fetal Intestinal Organoids. J. Vis. Exp. (153), e60470, doi:10.3791/60470 (2019).

\section{Abstract}

At the end of the suckling period, many mammalian species undergo major changes in the intestinal epithelium that are associated with the capability to digest solid food. This process is termed suckling-to-weaning transition and results in the replacement of neonatal epithelium with adult epithelium which goes hand in hand with metabolic and morphological adjustments. These complex developmental changes are the result of a genetic program that is intrinsic to the intestinal epithelial cells but can, to some extent, be modulated by extrinsic factors. Prolonged culture of mouse primary intestinal epithelial cells from late fetal period, recapitulates suckling-to-weaning transition in vitro. Here, we describe a detailed protocol for mouse fetal intestinal organoid culture best suited to model this process in vitro. We describe several useful assays designed to monitor the change of intestinal functions associated with suckling-to-weaning transition over time. Additionally, we include an example of an extrinsic factor that is capable to affect suckling-to-weaning transition in vivo, as a representation of modulating the timing of suckling-to-weaning transition in vitro. This in vitro approach can be used to study molecular mechanisms of the suckling-to-weaning transition as well as modulators of this process. Importantly, with respect to animal ethics in research, replacing in vivo models by this in vitro model contributes to refinement of animal experiments and possibly to a reduction in the use of animals to study gut maturation processes.

\section{Video Link}

The video component of this article can be found at https://www.jove.com/video/60470/

\section{Introduction}

In many mammalian species, including mice and men, the neonatal intestine has several features that are distinct from the fully mature intestinal epithelium. These features facilitate neonatal enterocytes to digest and absorb milk, which contains high fat and low carbohydrates, with lactose as the major carbohydrate. The brush border of the neonatal intestinal epithelial cells express the disaccharidase lactase-phlorizin hydrolase (Lct) ${ }^{1}$ to digest the milk disaccharide lactose. After the suckling period, enterocytes adapt to digest solid food that is rich in complex carbohydrates and low in fat. This is manifested by a switch in brush border disaccharidase expression from lactase to sucrase-isomaltase (Sis) and trehalase (Treh), which can digest more complex carbohydrates present in solid food ${ }^{2}$. Another metabolic switch is related to the low concentration of arginine in milk. To provide for the need for arginine, neonatal enterocytes express the rate limiting enzyme in arginine biosynthesis, argininosuccinate synthase-1 (Ass1), to synthetize arginine ${ }^{3}$. In contrast, adult enterocytes express arginase 2 (Arg2), an enzyme capable of catabolizing arginine that is abundant in solid foods. Furthermore, the neonatal intestinal epithelium expresses the neonatal Fc receptor for immunoglobulins ( $\mathrm{FCRn}$ ), which mediates absorption of the maternal IgG from the milk into the circulation/bloodstream ${ }^{4}$. The expression of FcRn declines significantly during the suckling-to-weaning transition ${ }^{5}$. In mice, maturation of Paneth cells occurs postnatally, coincidently with the formation and maturation of crypts, and is characterized by expression of antimicrobial peptides lysosome (Lyz) and defensins ${ }^{6}$.

All these changes are part of the suckling-to-weaning transition, a process occurring gradually after birth to one month of age in mice, when the intestinal epithelium reaches its mature adult state. Suckling-to-weaning transition is intrinsically regulated and developmentally set in the gut tube. Transcription factor B lymphocyte-induced maturation protein-1 (Blimp-1) plays a key role in this intrinsic maturation process ${ }^{7}$. Blimp-1 is highly expressed in neonatal epithelium, while its expression decreases and is lost during the suckling-to-weaning transition and therefore can serve as a reliable marker of neonatal intestinal epithelium. Despite being an intrinsic process, the suckling-to-weaning transition can be modulated by external factors. For example, the synthetic analogue of cortisol, dexamethasone, is known to accelerate gut maturation in vivo ${ }^{8,9}$.

Current in vitro models used to study intestinal epithelial maturation including the suckling-to-weaning transition, utilize adult epithelial cell lines and/or adult organoids which bear characteristics of adult intestinal epithelium. We have recently demonstrated that primary intestinal epithelial cells isolated from the late fetal intestine mature and recapitulate the suckling-to-weaning transition when growing in vitro as organoids ${ }^{10}$. We 
further showed that this gut maturation process in vitro occurs at the same pace as in vivo. Finally, we used dexamethasone to accelerate the maturation process in the same fashion described for in vivo studies.

Here, we outline a precise protocol for the isolation and culture of mouse late fetal intestinal organoids. We describe the preferred way of collecting samples for prolonged organoid culture and methods to monitor suckling-to-weaning transition in vitro. This protocol can be used for in vitro studies of intestinal epithelial maturation and modulators of this process and results in higher throughput, increased quality and translational value of the data and a reduction of animal use.

\section{Protocol}

This study was conducted in accordance with institutional guidelines for the care and use of laboratory animals established by the Ethic Committee for Animal Experimentation of the University of Amsterdam in full compliance to the national legislation on animal research following the European directive 2010/63/EU for the use of animals for scientific purposes (ALC312).

\section{Isolation of fetal small intestinal organoids}

1. Sacrifice the E18-E20 fetuses by decapitation with surgical scissors, according to the approved ethical regulations.

2. Immediately after euthanasia, carefully cut open the lower abdomen of the fetus with intestinal scissors and remove the whole intestine. NOTE: Isolation must be performed with intestines between E18 and E20 of gestation in order to achieve the proper suckling-to-weaning transition and maturation in vitro.

3. With two small forceps, stretch the intestine. Using the stomach and the appendix as guides, cut apart the proximal and distal part of the small intestine. NOTE: If dissection is done carefully, it is possible to isolate the colon as well. Cut it apart using appendix as guide (Figure 1).

4. Make the intestine open longitudinally: fix the intestine using a razor blade placed lengthwise and pull the intestine by sliding forceps (one arm of the forceps on each side of the razor blade) along the razor blade. Subsequently, cut the opened intestine in $1 \mathrm{~cm}$ pieces.

5. Prepare two $50 \mathrm{~mL}$ tubes with $10 \mathrm{~mL}$ of ice cold phosphate-buffered saline (PBS). Transfer the proximal and distal part of the small intestine (and the colon if applicable) separately to the tubes. Keep the tubes on ice while dissecting the intestines of additional mice. Collect all intestinal parts of one litter together in the same tube.

NOTE: One litter has usually 6-10 fetuses. The intestines of all fetuses must be combined. This amount should be enough to yield $4-8$ wells with organoids, in a 48-well plate, per intestinal part.

6. Proceed with organoid isolation as previously described ${ }^{11}$. In short, wash intestinal pieces with ice-cold PBS, incubate with 2 mM ethylenediaminetetraacetic acid (EDTA) for 30 minutes at $4{ }^{\circ} \mathrm{C}$, dissociate the crypts from the tissue by harshly washing the pieces with ice cold PBS $+10 \%$ fetal calf serum (FCS), filter using a $70 \mu \mathrm{m}$ cell strainer and centrifuge to collect the crypts at $150 \times \mathrm{g}$ for $5 \mathrm{~min}$ at $4{ }^{\circ} \mathrm{C}$.

7. Plate 4 to 8 wells with crypts in extracellular matrix gel, depending on the pellet size, in a warm 48 -well plate. Use $20 \mu \mathrm{L}$ of crypts suspended in extracellular matrix gel per well. Let extracellular matrix gel solidify in a $37^{\circ} \mathrm{C}$ incubator for $10 \mathrm{~min}$.

NOTE: Considering that only $40 \%$ to $50 \%$ of isolated crypts form organoids, aim for a density of 250 to 300 organoids per well. First add less extracellular matrix gel than needed. Look under the microscope after platting the first well to analyze whether the density of the isolated crypts is ideal. If necessary, add more extracellular matrix gel.

8. In the meantime prepare ENR medium: $14 \mathrm{~mL}$ of Advanced Dulbecco's Modified Eagle Medium/Ham's F-12 (DMEM/F12) 1:1 ++ + (supplemented with 4-(2-hydroxyethyl)-1-piperazineethanesulfonic acid (HEPES) 1x, L-glutamine $0.01 \mathrm{M}$ and $0.2 \mathrm{U} / \mathrm{mL}$ Penicillin/ Streptomycin), $4 \mathrm{~mL}$ of Noggin-conditioned media, $2 \mathrm{~mL}$ of Rspondin-conditioned media, $400 \mu \mathrm{L}$ of B27 supplement $1 \mathrm{x}, 200 \mu \mathrm{L}$ of $\mathrm{N} 2$ supplement $1 \mathrm{x}, 50 \mu \mathrm{L}$ of $1.25 \mathrm{mM}$-Acetylcysteine, $20 \mu \mathrm{L}$ of $0.05 \mu \mathrm{g} / \mathrm{mL}$ Epidermal Growth Factor (EGF). NOTE: When culturing colon organoids, supplement with $50 \%$ Wnt conditioned media.

9. Add $250 \mu \mathrm{L}$ of ENR medium per well.

\section{Culturing of fetal organoids}

1. Change medium of the cultures 3 times per week. Maturation of the organoids is achieved after 1 month of culture.

2. At day 3 after each passage, count the number of spheroids and organoids using an optical microscope. Quantify at least 3 wells per condition and all the organoids present in each well.

NOTE: Number of spheroids reduces with time, while the number of organoids increases (Figure 2).

3. Passage the organoids once a week by mechanical dissociation as described below.

1. Remove medium and add $1 \mathrm{~mL}$ of ice-cold Advanced DMEM/F12 1:1 +++. Collect all extracellular matrix gel with organoids into a 15 $\mathrm{mL}$ tube. Use a $200 \mu \mathrm{L}$ tip on top of a $1000 \mu \mathrm{L}$ filter tip and pipette up and down 20 times to disrupt the organoids.

2. Centrifuge at $150 \times g$ for $5 \mathrm{~min}$ at $4{ }^{\circ} \mathrm{C}$. Discard the supernatant and resuspend the pellet in $20 \mu \mathrm{L}$ of extracellular matrix gel per well. Usually, fetal organoids can be expanded in a 1:2 ratio.

3. Let extracellular matrix gel solidify for 5-10 min. Add $250 \mu \mathrm{L}$ of ENR medium per well.

\section{Maturation analysis at RNA and protein level}

1. Analyze culture every 3 days after each passage, for a period of 1 month (i.e., time in which complete maturation is achieved) (Figure 3 ). NOTE: Fetal organoid culture is dynamic (Movie 1) and to avoid variation from the normal regeneration of organoids after mechanical disruption, it is necessary to always collect samples at the same day after passage.

2. RNA isolation 
1. Collect 3 wells of organoids using $200 \mu \mathrm{L}$ of RNA lysis buffer for each well supplemented with $2 \mu \mathrm{L}$ of $\beta$-mercaptoethanol. After adding RNA lysis buffer $+\beta$-mercaptoethanol to the well, make sure all extracellular matrix gel with organoids is transferred to a RNase-free $1.5 \mathrm{~mL}$ tube.

2. Vortex vigorously and keep at $-80^{\circ} \mathrm{C}$ for no longer than 1 month. Isolate RNA using commercially available silica spin column kits.

3. To increase RNA quality, after washing steps add $500 \mu \mathrm{L}$ of $80 \% \mathrm{EtOH}$ and gently mix by inverting the column. Centrifuge for 2 min at $11,000 \times g$ to dry the column completely.

NOTE: Make sure to wash the inside of the lid of the tube with the $80 \% \mathrm{EtOH}$ by flicking the tube upside down three to five times to get rid of all traces of guanidine thiocyanate.

4. To increase RNA yield, wait 1-2 min after applying RNase-free water before centrifuge. Re-elute RNA by applying the first flow-through eluate to the column a second time.

NOTE: Isolated RNA quality is sufficient for use in genome-wide expression analysis. Check whether RNA integrity number is above 8.

\section{Protein isolation}

1. Collect 5 wells of organoids using $250 \mu \mathrm{L}$ of ice-cold cell recovery solution into one $15 \mathrm{~mL}$ tube. Incubate for at least $30 \mathrm{~min}$ on ice to dissolve the extracellular matrix gel (this will reduce protein contribution from extracellular matrix gel).

2. Wash with ice-cold PBS. Add $250 \mu \mathrm{L}$ of cell lysis buffer and store at $-80^{\circ} \mathrm{C}$. NOTE: After sonication, samples can be used to detect enzyme activity or for Western Blots.

\section{Immunostaining}

1. Collect 2 wells of organoids using $250 \mu \mathrm{L}$ of ice-cold cell recovery solution into a $15 \mathrm{~mL}$ tube. Incubate for at least $30 \mathrm{~min}$ on ice to dissolve the extracellular matrix gel (this will reduce staining background). Wash with ice-cold PBS.

2. Fix the organoids using $500 \mu \mathrm{L}$ of of $4 \%$ paraformaldegyde (PFA) for $1 \mathrm{~h}$ at $4{ }^{\circ} \mathrm{C}$. Wash with ice-cold PBS. Proceed to whole-organoid immunofluorescence or to paraffin embedding, according to published protocols ${ }^{12}$.

NOTE: Use a plastic Pasteur pipette to handle the organoids. This will avoid disruption of its structure.

\section{Effect of extrinsic factor (dexamethasone as an example) on organoid maturation process}

1. On day one of culture, add $0.01 \mathrm{M}$ dexamethasone to the organoids. Incubate the organoids with dexamethasone during the whole month of culture by adding new dexamethasone every time medium is changed.

2. Gene expression analysis

1. Isolate RNA as described above. Synthesize, at the same time, cDNA of all samples to be compared (treated and untreated). Proceed with preferred qRTPCR method.

2. Use GeNorm to identify the two most stable reference gene every time a new treatment is used on the fetal organoids. Use the geometric mean of the two chosen reference genes for relative expression calculations.

NOTE: Suggestions of reference genes to test for mouse fetal organoids: Cyclophilin, Gapdh, Bactin, 36b4, Hprt, Rpl4, Rpl32, Ppib and Tbp.

3. To investigate how a certain extrinsic factor affects postnatal mouse fetal maturation, all the following genes should be evaluated.

1. Check whether fetal markers lactase ( $L c t)$, argininosuccinate synthase 1 (Ass1), B lymphocyte-induced maturation protein 1 $(B l i m p-1)$ and neonatal Fc receptor $(F c R n)$ decrease in expression during the first two weeks of culture and are absent for the remaining culture time (Figure 4C). Analyze whether this pattern is altered.

2. Check whether adult markers sucrase-isomaltase (Sis), arginase 2 (Arg2), trehalase (Treh) and lysozyme (Lyz) increase in expression after two weeks of culture (Figure 4D). Analyze whether this pattern is altered by the external factor. NOTE: Dexamethasone is an external factor that can accelerate the maturation of the fetal organoids and can be used as a positive control in all experiments aimed to test other extrinsic factors.

\section{Enzyme activity analysis}

1. Isolate protein as described above. Detect intestinal disaccharidases activity according to protocols published by Dahlqvist and Messer ${ }^{13,14}$

2. Prepare $0.625 \mathrm{M}$ maleic-buffer $\mathrm{pH} 6.0$ (keep for 3 months at $4{ }^{\circ} \mathrm{C}$ ). Using this buffer, prepare $0.05 \mathrm{M}$ lactose (add phydroxymercuribenzoate sodium as stabilizer to inhibit lysosomal p-galactosidase activity); $0.05 \mathrm{M}$ maltose; $0.05 \mathrm{M}$ sucrose and $0.05 \mathrm{M}$ trehalose.

NOTE: All these solutions can be kept for 5 days at $4{ }^{\circ} \mathrm{C}$. Keep on ice while preparing assay.

3. Prepare assay standards by diluting $5.56 \mathrm{M}$ glucose solution with ultrapure water to obtain solutions with the following concentrations: $0.125 \mathrm{M} ; 0.1 \mathrm{M} ; 0.075 \mathrm{M} ; 0.05 \mathrm{M}$ and $0.025 \mathrm{M}$.

NOTE: solution stable for at least 3 months at $4{ }^{\circ} \mathrm{C}$.

4. Incubate in a 96 -well plate for $60 \mathrm{~min}$ at $37^{\circ} \mathrm{C}$ :

- $30 \mu \mathrm{L}$ of organoid lysate with $30 \mu \mathrm{L}$ of lactose

- $30 \mu \mathrm{L}$ of organoid lysate with $30 \mu \mathrm{L}$ of sucrose

- $30 \mu \mathrm{L}$ of ten times diluted organoid lysate with $30 \mu \mathrm{L}$ of maltose

- $30 \mu \mathrm{L}$ of five times diluted organoid lysate with $30 \mu \mathrm{L}$ of trehalase

- $30 \mu \mathrm{L}$ of undiluted organoid lysate with $30 \mu \mathrm{L}$ of maleic acid, as sample background

- $30 \mu \mathrm{L}$ of each glucose standard

- $30 \mu \mathrm{L}$ of ultrapure water, as blank

- $30 \mu \mathrm{L}$ of positive control (optimize dilution; lysed fetal intestinal tissue can be used as control for lactase activity while lysed adult intestinal tissue can be used as control for sucrase, maltase and trehalase)

NOTE: Dilution of samples should be made with cell lysis buffer. Keep the samples and plate on ice while preparing the assay. 
5. To determine the amount of glucose produced by the enzymes present in the organoid lysate after incubation with their respective substrates, add quickly $200 \mu \mathrm{L}$ of PGO-color solution and measure absorbance at $450 \mathrm{~nm}$ every 5 min for $30 \mathrm{~min}$ at $37^{\circ} \mathrm{C}$. NOTE: Make PGO-color solution fresh. Use $10 \mathrm{U} / \mathrm{mL}$ glucose-oxidase, $2 \mathrm{U} / \mathrm{mL}$ peroxidase and $7.88 \mathrm{mmol} / \mathrm{L}$ o-dianisidine in $0.5 \mathrm{~mol} / \mathrm{L}$ Tris- $\mathrm{HCl}$ buffer at $\mathrm{pH}$ 7.0. Solution should be at room temperature when added to the plate.

6. Calculate activity according to glucose standard and correct for total amount of protein (determined by bicinchoninic acid assay (BCA)). Enzyme activity should be expressed as $\mu \mathrm{M}$ glucose $/ \mu \mathrm{g}$ protein $\cdot \mathrm{min}^{-1}$ (Figure $5 \mathrm{~B}$ ).

NOTE: Measure arginase activity using a commercially available arginase activity assay kit.

\section{Representative Results}

\section{Prolonged culture of fetal intestinal epithelial cells}

The protocol for mimicking suckling-to-weaning transition in vitro depends on correct handling of fetal organoids during prolonged culture. Proximal and distal intestine isolated from E18-E20 mouse fetuses are separated as presented in (Figure 1). Upon isolation, epithelial cells are seeded in extracellular matrix gel domes (Figure 2). It typically takes four passages and approximately 28-30 days of culture for fetal organoids to mature to the adult state. During this time, cells at various stages of maturation can be collected (Figure 3 ).

\section{Representative downstream analysis of suckling-to-weaning transition in vitro}

To confirm that isolated fetal organoids are distinctly proximal or distal, compare the expression level of proximal markers One cut domain family member 2 (Onecut2) and GATA binding protein 4 (Gata4) and distal markers Fatty acid binding protein 6 (Fabp6) and Guanylate Cyclase Activator 2A (Guca2a) between both proximal and distal organoid cultures (Figure 4A,B). Suckling-to-weaning transition in vitro can be monitored by two sets of genes: fetal (Figure 4C) and adult markers (Figure 4D). Fetal markers should gradually decrease during the course of the culture, while the expression of adult markers should gradually increase (Figure 4C,D).

\section{Using extrinsic factor as a modifier of sucking-to weaning transition in vitro}

In this protocol dexamethasone a synthetic glucocorticoid, was used as an example of extrinsic factor capable of modifying suckling-to-weaning transition in vitro. Representative data in Figure $\mathbf{5}$ suggests that effects of extrinsic factors are best to be determined by multiple assays, as they do not necessarily ought to be genomic. For example, in the case of sucrase-isomaltase both RNA and protein expression are induced with dexamethasone (Figure 5A) whereas trehalase expression is only changed at the protein level. (Figure 5B).

A

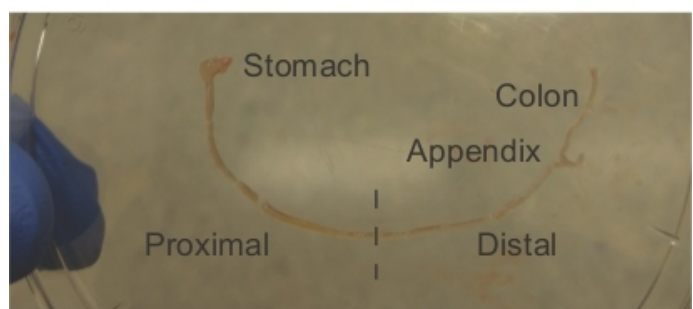

Figure 1: Isolation of mouse fetal small intestine. (A) Photograph of dissected and stretched fetal gut, including stomach, proximal and distal small intestine, appendix and colon. Black line indicates where gut should be cut to divide the proximal and the distal small intestine. Please click here to view a larger version of this figure. 


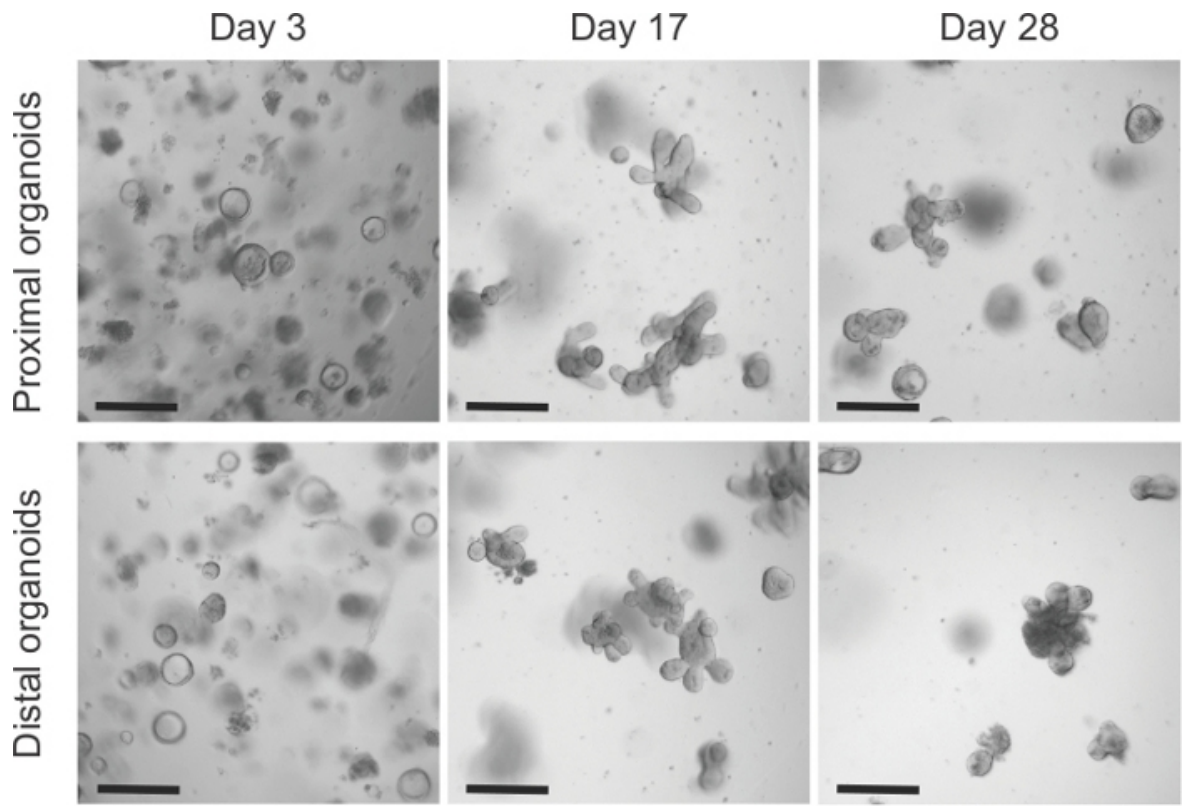

Figure 2: Representative microscopic images of proximal and distal fetal organoid culture at day 3, day 17 and day 28 of culture. All images were obtained at day 3 after passage and show the decrease in the number of spheroids overtime. Scale bar: $500 \mu \mathrm{m}$. Please click here to view a larger version of this figure.

Movie 1: Representative video showing fetal organoid culture dynamics, from day $\mathbf{4}$ to $\mathbf{6}$ of culture. Please click here to view this video. (Right-click to download.)

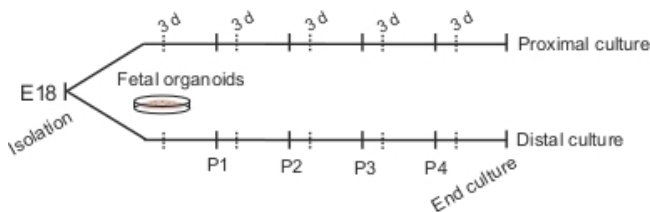

Figure 3: Schematic representation of organoid collection for analysis of gut maturation over time. Proximal and distal fetal organoid cultures should be cultured for one month and passaged every week. Samples for maturation analysis should be collected 3 days after isolation and every 3 days after each passage. Please click here to view a larger version of this figure.

A

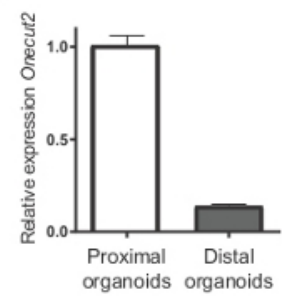

C

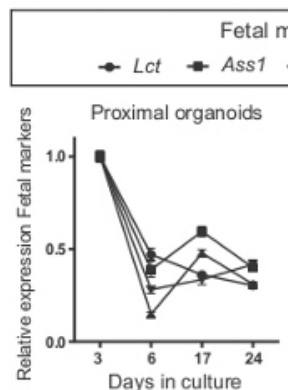

B

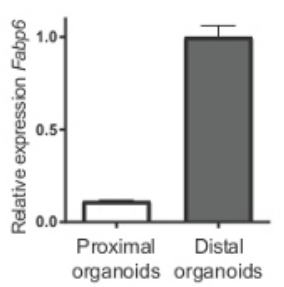

D

Adult markers
$\rightarrow$ Sis - Arg $2+$ Treh $*$ Lyz
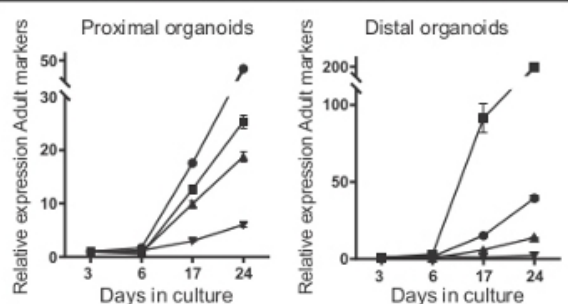
Gata4 are mainly expressed in the proximal organoid culture while (B) distal markers Fabp6 and Guca2a are mostly expressed in the distal organoid culture. (C) Fetal markers Lct, Ass1, Blimp-1 and FcRn decrease and (D) adult markers Sis, Arg2, Treh and Lyz increase over time in both proximal and distal organoid cultures. Error bars represent SEM. Please click here to view a larger version of this figure. 
A

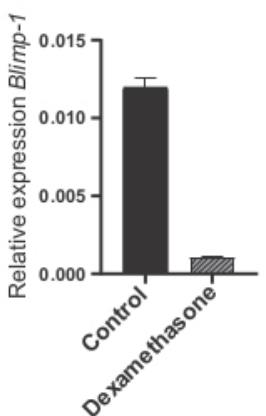

B

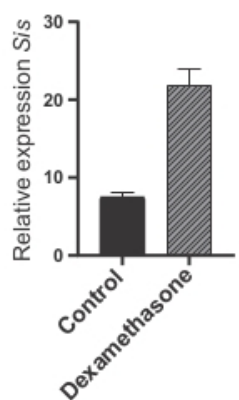

C

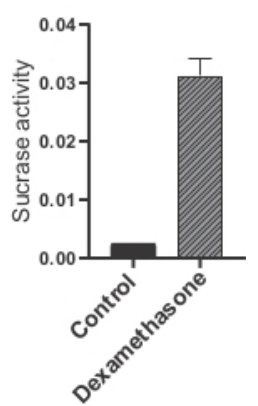

Figure 5: External factor dexamethasone can modulate the maturation of fetal organoids. (A) Gene expression of fetal marker Blimp-1 is decreased at day 12 of culture in dexamethasone treated organoids compared to control organoids, while both gene expression (B) and enzyme activity $(\mathrm{C})$ of adult marker sucrase-isomaltase (Sis) is increased. Please click here to view a larger version of this figure.

\section{Discussion}

This protocol describes culturing of late fetal intestinal organoids for prolonged time to mimic suckling-to-weaning transition in vitro. The process of maturation equals the pace in vivo and is completed after one month in culture. Downstream analysis of this culture using quantitative RNA and protein techniques are detailed.

In this protocol, primary intestinal cells from E18-E20 mouse embryos are used. The developmental stage of primary mouse cells used to generate organoids for this protocol is particularly important. Using earlier developmental stage will result in generation of intestinal spheroids that maintain their specific fetal gene expression over a prolonged period of time with limited transition to adult organoids ${ }^{15,16}$. Only late fetal stage spheroids are capable in transiting to adult organoids in vitro ${ }^{10}$. To maximize the window of opportunity with respect to impact of extrinsic factors on gut maturation, intestines from late fetal stage are recommended and not intestines from born pups that have been exposed to microbes and mother milk. It is reported that certain bacterial metabolites and milk components can act as modifiers of the maturation process ${ }^{17}$.

To obtain sufficient amounts of cells to maintain the culture for one month to study the whole maturation from birth up to adulthood, while collecting the samples for downstream analyses, intestines from 6-8 embryos should be used as starting material. It is preferred to use embryos from the same developmental stage for generating the culture. We do not recommend pooling different litters as slight differences in developmental stage can influence expression of the maturation genes.

The protocol described here accounts for organoid generation from the proximal and distal small intestine to maintain developmental features of different segments of the gut. As an alternative, whole intestine can be used to investigate overall maturation with respect to the increase/ decrease expression of the specific markers. In the latter case, fewer embryos could be used to isolate intestinal cells for starting culture.

This protocol is developed using three-dimensional organoid cultures. As organoids undergo dynamic growth in the culture, it is important to collect samples for downstream analyses at the same time point after passaging. In this protocol, we have selected day 3 after passage, as it represents the medium time between two splits at which organoids contain robust buds and little to no cell death. An alternate time point after passaging can be used, but it should be consistent during the whole experiment. We do not recommend growing organoids for more than 7 days after a passage, as increase of death cells in the organoid lumen can affect the results.

In our experiments, we have used dexamethasone as an example of an extrinsic factor that is shown and best studied in literature to accelerate intestinal maturation in vivo ${ }^{9,18}$. Dexamethasone exerts its effects via both genomic and non-genomic routes. For example, on the level of genomic regulation, a precocious increase of Sis mRNA levels can be observed. On a non-genomic level, we observe alterations in the activity of digestive enzymes such as trehalase. Both are in accordance with described specific aspects of dexamethasone on sucrase gene activation and non-genomic activating effect on intestinal brush border enzymes observed in vivo ${ }^{19}$. The fact that extrinsic factors, like synthetic glucocorticoids, can modulate certain aspects of suckling-to-weaning transition in the organoid culture, similarly to that described in vivo, further establishes the mouse fetal intestinal organoids as a model for the investigation of different kind of modulators of gut maturation.

Even though the morphological maturation of human intestinal epithelium is completed in utero at gestational stage of 22 weeks, the intestinal barrier function matures till childhood in a close relationship with the type of feeding, development of microbiota and immune response. Due to the limited availability of human tissues at these developmental stages, the translational value of in vitro murine model lies in the possibility of high throughput screens of factors capable of modulating intestinal maturation, a process conserved among suckling mammals.

Importantly, with respect to animal ethics in research, this model can contribute to refinement of animal experiments as it does not include interventions performed on animals. The number of animals can be further reduced by redesigning research questions to one or two time points of culture which will allow testing of multiple components within one culture.

Authors declare nothing to disclose. 


\section{Acknowledgments}

None.

\section{References}

1. Henning, S. J. Postnatal development: coordination of feeding, digestion, and metabolism. American Journal of Physiology. 241 (3), G199-214 (1981).

2. Krasinski, S. D. et al. Transcriptional regulation of intestinal hydrolase biosynthesis during postnatal development in rats. American Journal of Physiology. 267 (4 Pt 1), G584-594 (1994).

3. Hurwitz, R., Kretchmer, N. Development of arginine-synthesizing enzymes in mouse intestine. American Journal of Physiology. 251 (1 Pt 1 ), G103-110 (1986).

4. Rath, T. et al. The immunologic functions of the neonatal Fc receptor for IgG. Journal of Clinical Immunology. 33 Suppl 1 S9-17 (2013).

5. Martin, M. G., Wu, S. V., Walsh, J. H. Ontogenetic development and distribution of antibody transport and Fc receptor mRNA expression in rat intestine. Digestive Diseases and Sciences. 42 (5), 1062-1069 (1997).

6. Bry, L. et al. Paneth cell differentiation in the developing intestine of normal and transgenic mice. Proceedings of the National Academy of Sciences of the United States of America. 91 (22), 10335-10339 (1994).

7. Muncan, V. et al. Blimp1 regulates the transition of neonatal to adult intestinal epithelium. Nauret Communications. 2 452 (2011).

8. Beaulieu, J. F., Calvert, R. Influences of dexamethasone on the maturation of fetal mouse intestinal mucosa in organ culture. Comparative Biochemistry and Physiology A Comparative Physiology. 82 (1), 91-95 (1985).

9. Nanthakumar, N. N., Henning, S. J. Ontogeny of sucrase-isomaltase gene expression in rat intestine: responsiveness to glucocorticoids. American Journal of Physiology. 264 (2 Pt 1), G306-311 (1993).

10. Navis, M. et al. Mouse fetal intestinal organoids: new model to study epithelial maturation from suckling to weaning. EMBO Reports. 20 (2) (2019).

11. Sato, T. et al. Single Lgr5 stem cells build crypt-villus structures in vitro without a mesenchymal niche. Nature. 459 (7244), $262-265$ (2009).

12. Van Lidth de Jeude, J. F., Vermeulen, J. L., Montenegro-Miranda, P. S., Van den Brink, G. R., Heijmans, J. A protocol for lentiviral transduction and downstream analysis of intestinal organoids. Journal of Visualized Experiments. (98) (2015).

13. Dahlqvist, A. Assay of intestinal disaccharidases. Scandinavian Journal of Clinical and Laboratory Investigation. 44 (2), 169-172 (1984).

14. Messer, M., Dahlqvist, A. A one-step ultramicro method for the assay of intestinal disaccharidases. Anal Biochemistry. 14 (3), $376-392$ (1966).

15. Fordham, R. P. et al. Transplantation of expanded fetal intestinal progenitors contributes to colon regeneration after injury. Cell Stem Cell. 13 (6), 734-744 (2013).

16. Mustata, R. C. et al. Identification of Lgr5-independent spheroid-generating progenitors of the mouse fetal intestinal epithelium. Cell Reports. 5 (2), 421-432 (2013).

17. Holscher, H. D., Bode, L., Tappenden, K. A. Human Milk Oligosaccharides Influence Intestinal Epithelial Cell Maturation In Vitro. Journal of Pediatric Gastroenterology and Nutrition. 64 (2), 296-301 (2017).

18. Solomon, N. S., Gartner, H., Oesterreicher, T. J., Henning, S. J. Development of glucocorticoid-responsiveness in mouse intestine. Pediatric Research. 49 (6), 782-788 (2001).

19. Kedinger, M., Simon, P. M., Raul, F., Grenier, J. F., Haffen, K. The effect of dexamethasone on the development of rat intestinal brush border enzymes in organ culture. Developmental Biology. 74 (1), 9-21 (1980). 